\section{Comparing Binary Image Analysis Measurements - Euclidean Geometry, Centroids and Corners}

\author{
Dennis W. Hetzner \\ Timken, Co. Canton, Ohio \\ dennis.hetzner@timken.com
}

\section{Introduction}

During the past several years, ASTM Subcommittee E04.14 on Quantitative Metallography has been developing an Image Analysis (I/A) methodology to determine the degree of circularity, roundness or sphericity of graphite nodules in ductile cast iron. In the as-polished condition, ideally the iron nodules should appear as dark circular objects in a light gray matrix, Figure 1 . When performing Image Analysis measurements for these types of materials, complex gray image procedures should not be required to properly threshold the images to obtain an acceptable binary image on which to perform the measurements.

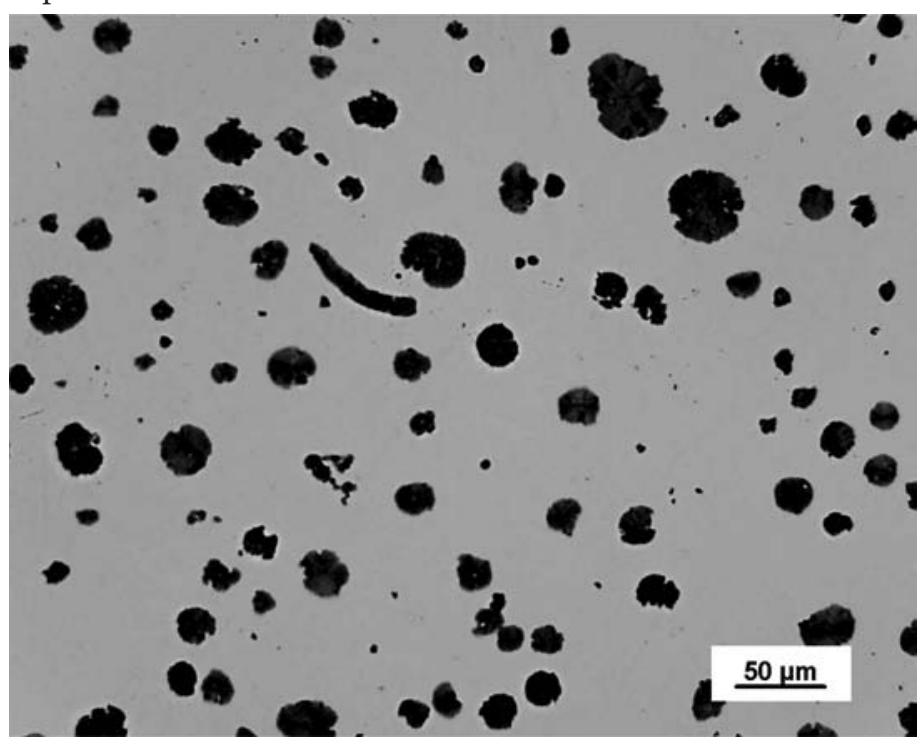

Figure 1. Gray image showing various morphologies of ductile iron particles

During the development of the standard, it became evident that specific tolerances on the magnification used to perform the measurements or, more precisely, the number of pixels in the objects to be measured in the binary images, needed to be clearly specified. This was because during a preliminary test program, it was discovered that differences in the magnification used for the analysis could affect the results of the measurements.

As development of the standard progressed, not only was the magnification used for the analysis found to be important, but differences in the way I/A systems made measurements on binary images appeared to be of equal importance. Thus, some method of determining how different I/A systems made basic measurements was required.

\section{Experimental Procedures}

Initially, using a graphical interface, a preliminary test pattern consisting of a series of circles of known diameters was created. Members of Subcommittee E04.14 measured the circles with several different I/A systems, and it was discovered that there was a wide variation in the resulting measurements. This led to the creation of a second test image. The second test image consisted of the fol- lowing series of filled objects:

1. Straight lines parallel to the $\mathrm{x}$-axis of the I/A system having pixel lengths of 10, 20, 30, 40 and 50 .

2. Rectangles having sides parallel to the principal axes of the I/A system and having dimensions of $(3 \times 4),(6 \times 8),(9 \times 12)$, $(12 \times 16)$ and $(15 \times 20)$ pixels.

3. Inscribed circles having pixel diameters of 4, 8, 16, 32 and 64 .

4. A cosine rose having five leaves and a diameter of 200 pixels was included in the test pattern.

This test image was measured by four members of subcommittee E04.14 having different I/A systems. The same test pattern was later measured by several different manufacturers of I/A systems and software that attended the 2007 M\&M show in Ft. Lauderdale, Florida. Eventually, software packages from the following vendors were used in the evaluation: Buehler Ltd., Carl Zeiss, Clemex, Leica, NIH Image J, Olympus, Smart Imaging and Struers.

The test results to be described were based on measurements made from exactly the same binary image. For simplicity, the calibration of the systems was set so that 1 pixel was equal to $1.0 \mu \mathrm{m}$, and the calibration was the same in both the horizontal and vertical directions; i.e. $\mathrm{Cal} X=\mathrm{Cal} Y$. For each object in the test pattern, the area, longest Feret's diameter $\left(F_{\mathrm{Max}}\right)$, minimum Feret's diameter, perimeter and convex perimeter were measured.

\section{Results and Discussion}

Rectangles: The simplest object to consider first is the series of rectangles. As described, each of these rectangles is based on the ratio of sides having lengths of $3 \times 4$. Based on simple Euclidian geometry, the area of a $3 \times 4$ rectangle is 12 , the perimeter is 14 and using the Pythagorean theorem, the diagonal, or maximum Feret's diameter, is 5 . Similarly, for the $6 \times 8$ rectangle, the area would be 48 , the perimeter would be 28 and $F_{\text {Max. }}$ would be 10 , etc. When I/A measurements were performed by the various systems, the areas were exactly what would be calculated from simple Euclidean geometry. However, measurements of the other parameters showed considerable variations from the answers based on Euclidean geometry. For the $3 \times 4$ rectangle, the areas, maximum Feret's diameters and perimeters, as measured by the various systems, were as follows:

Table 1. Parameters for $3 \times 4$ rectangles measured by different I/A systems

\begin{tabular}{|c|c|c|c|}
\hline & Area & Length & Perimeter \\
\hline System & & & \\
\hline A & 12 & 4.50 & 10.00 \\
\hline B & 12 & 4.61 & 14.00 \\
\hline C & 12 & 3.61 & 10.00 \\
\hline D & 12 & 5.00 & 12.83 \\
\hline E & 12 & 5.00 & 11.66 \\
\hline F & 12 & 4.00 & 14.00 \\
\hline G & 12 & 4.95 & 12.17 \\
\hline H & 12 & 5.00 & 12.83 \\
\hline Min & 12 & 3.61 & 10.00 \\
\hline Max & 12 & 5.00 & 14.00 \\
\hline Average & 12 & 4.50 & 12.28 \\
\hline \% Diff. & 0 & 30.3 & 33.0 \\
\hline
\end{tabular}


In the above table, the letters $\mathrm{A}$ through $\mathrm{H}$ represent the different I/A systems used for the analysis. The term percent difference is defined as:

$$
\% \text { Difference }=\frac{(\text { Max }- \text { Min })}{\text { Mean }} \times 100
$$

This term is referred to as difference rather than error. That is because while the results are not the same for each system, the operators made no errors in performing the tests or the analysis. The differences are only due to the differences among the algorithms used to make the measurements.

Similarly, the maximum Ferret's diameters and perimeters for the different sized rectangles were found to be as follows:

Table 2. Differences among I/A parameters measured for various size rectangles

\begin{tabular}{|c|c|c|c|c|c|}
\hline \multicolumn{6}{|c|}{ Rectangles } \\
\hline $\boldsymbol{F}_{\text {Max. }}$ & $\mathbf{3 : 4}$ & $\mathbf{6 : 8}$ & $\mathbf{9 : 1 2}$ & $\mathbf{1 2 : 1 6}$ & $\mathbf{1 5 : 2 0}$ \\
\hline & & & & & \\
\hline Min. & 3.6 & 8.6 & 13.6 & 18.6 & 23.6 \\
\hline Max & 5.0 & 10.0 & 15.0 & 20.0 & 25.0 \\
\hline Mean & 4.5 & 9.5 & 14.5 & 19.5 & 24.5 \\
\hline & & & & & \\
\hline \% Diff. & 30.3 & 14.8 & 9.8 & 7.3 & 5.8 \\
\hline Perimeter & & & & & \\
\hline Min. & 10.0 & 24.0 & 38.0 & 52.0 & 66.0 \\
\hline Max & 14.0 & 28.0 & 42.0 & 56.0 & 70.0 \\
\hline Mean & 12.3 & 26.1 & 40.1 & 54.1 & 68.1 \\
\hline & & & & & \\
\hline \% Diff. & 33.0 & 15.3 & 10.0 & 7.4 & 5.9 \\
\hline
\end{tabular}

The data and associated graph, Figure 2, clearly indicate that as the number of pixels in the rectangles increases, the difference among the systems used to perform the measurements decreases.

In order to explain the differences among the various systems, it is necessary to understand how the pixels composing the image

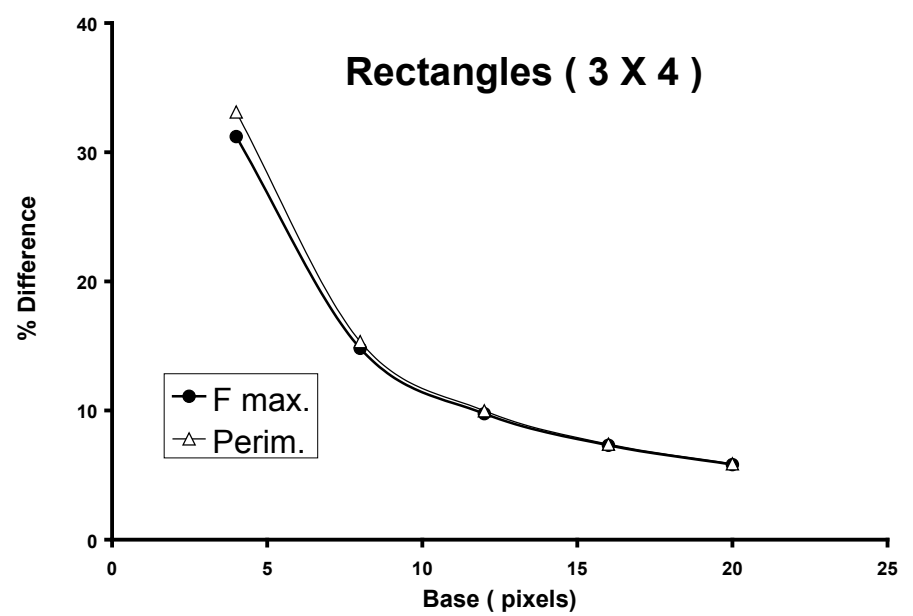

Figure 2. Difference among measured Image Analysis parameters with respect to rectangle size. are manipulated. First, considering simple Euclidean geometry, a $3 \times 4$ rectangle, based on the Pythagorean theorem would have a diagonal equal to 5, Figure 3.

\section{Outside Dimensions}
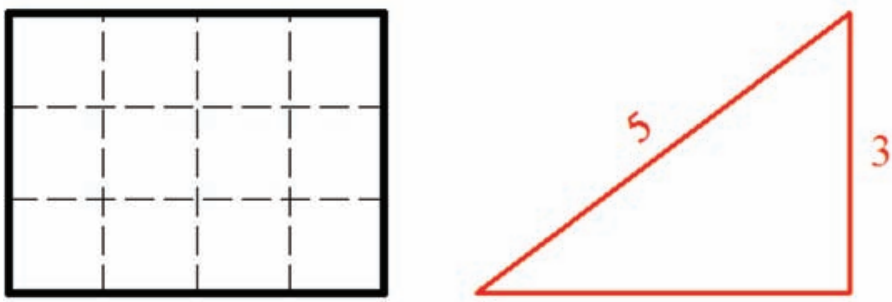

4

Figure 3. Euclidean Geometry: Dimensions of a $3 \times 4$ rectangle based on the outer dimensions.

However, as indicated by the dotted lines, each of the 12 pixels forming the rectangle contains a centroid, Figure 4 . Thus, in the $X$ direction, the distance between the centroids is 3 pixels. Similarly, the distance between the centroids in the $Y$ direction is only 2 pixels. By using the centroids and the Pythagorean theorem, the diagonal of the rectangle is $D^{2}=\left(F_{\text {Max. }}\right)^{2}=3^{2}+2^{2}=13$; hence, $F_{\text {Max. }}=3.61$. This explains the first difference among the various I/A systems.

\section{Centroids}
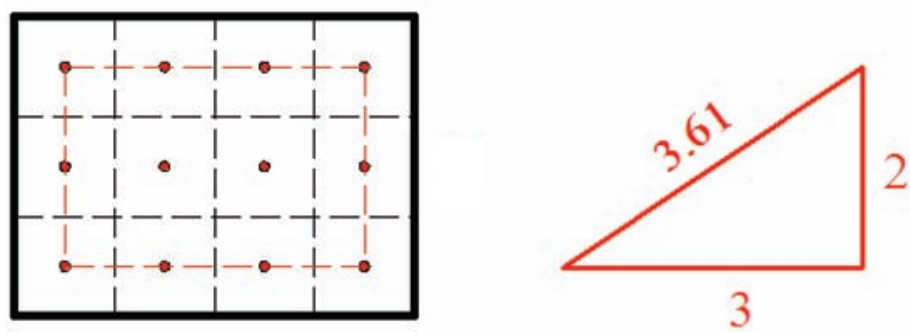

Figure 4. Dimensions of a $3 \times 4$ rectangle based on the centroids of the pixels forming the object.

Now, consider the perimeter of the $3 \times 4$ rectangle. Using simple Euclidean geometry, the lengths of each of the segments parallel to the $X$-axis are 4 pixels and the lengths of each of the segments parallel to the Y-axis are 3 pixels, Figure 3 . Thus, the simple perimeter of the $3 \times 4$ rectangle is $4+3+4+3=14$. However, now consider what can happen if the centroids are used for the measurement of perimeter. In this situation, again using simple Euclidean geometry, the lengths of the segments parallel to the $X$-axis are 3 pixels and the lengths of the segments parallel to the $Y$-axis are 2 pixels, Figure 4 . Thus the perimeter of the $3 \times 4$ rectangle based on pixel centroids is $3+2+3+2=10$.

These results account for the maximum and minimum values of the perimeter measurements; however, as indicated in Table 2 , other intermediate values also exist. To show how these other measurements are derived, the geometries of the corner pixels must be considered. In these tests, it was found that there were four different ways to analyze the corner pixels, Figure 5.

As shown by the circled pixel, Figure $5 \mathrm{a}$, the simplest method to account for the corner is to let it be equal to 2; that is, use the entire $X$ and $Y$ components of the corner pixel. The next level of refinement is to replace the two portions of the corner with a diagonal having a length of 1.41 pixels, Figure 5b. Finally, as was found in these tests, a shorter diagonal and a shorter portion of the corners can replace the corner pixel, Figures $5 \mathrm{c}$ and $\mathrm{d}$. Thus, different systems 

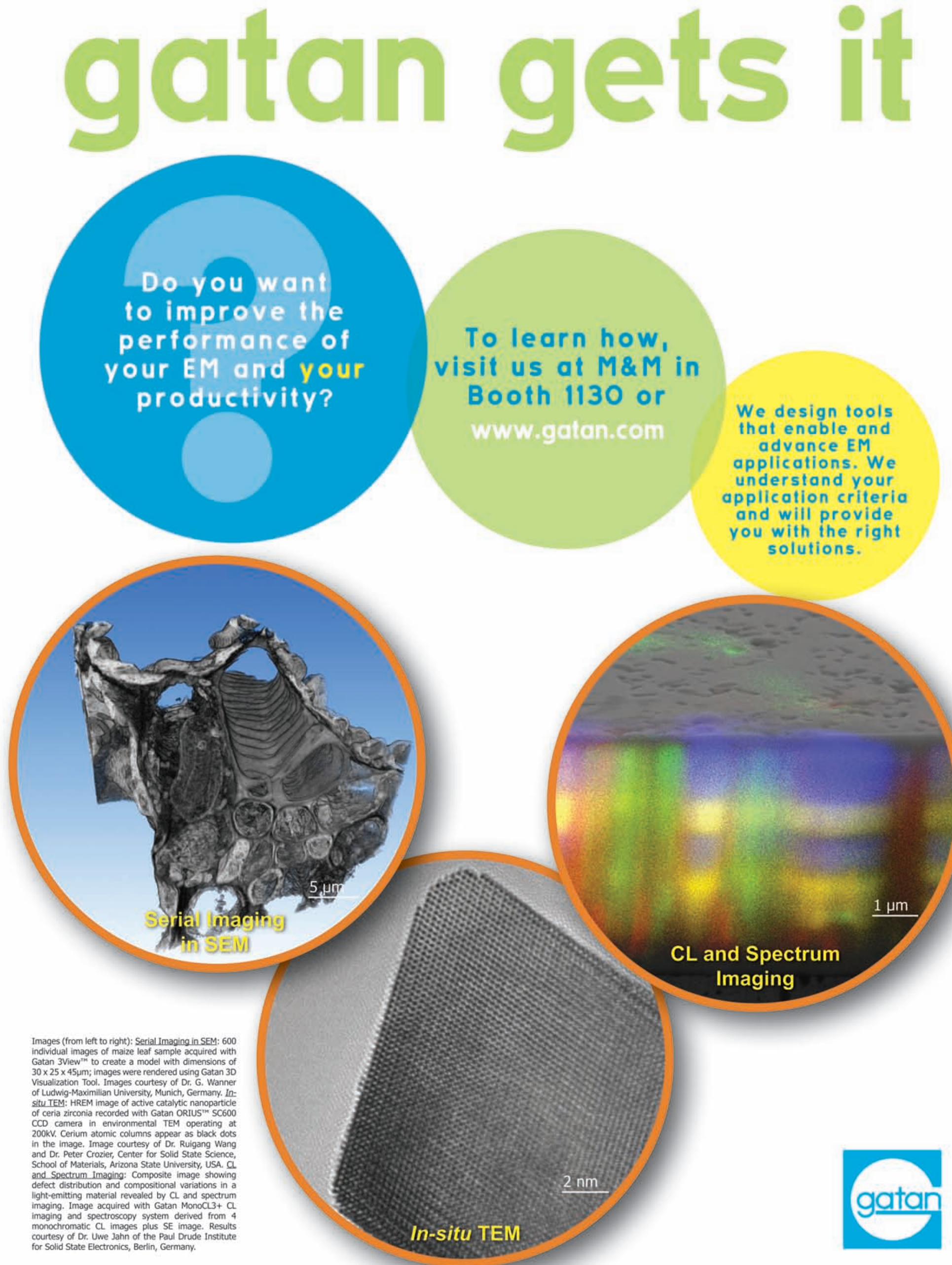


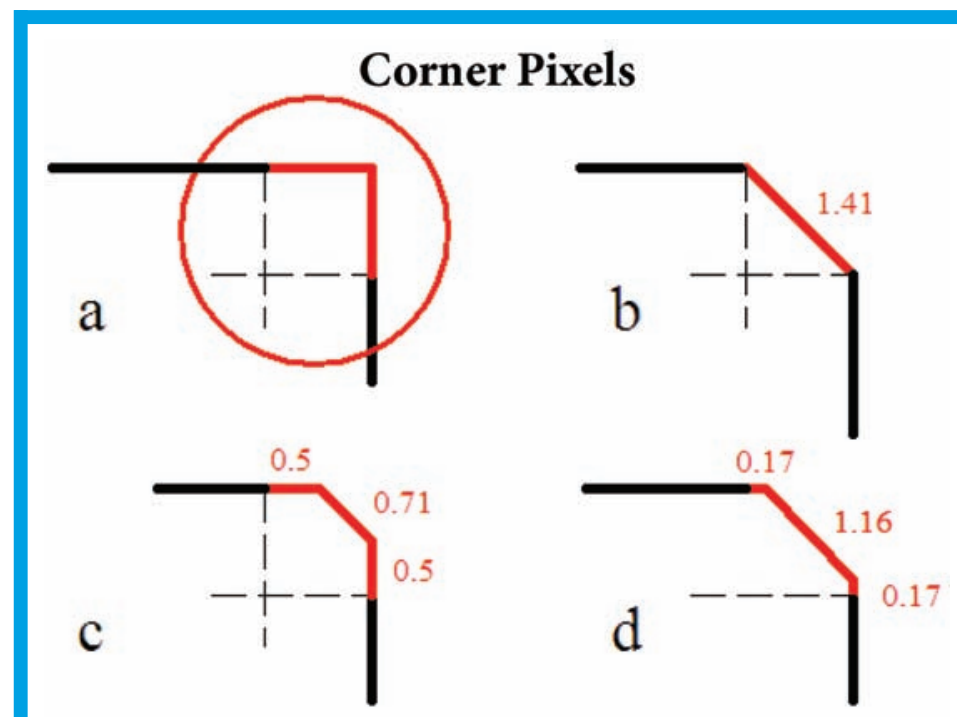

Figure 5. Geometrical methods for measuring the perimeter from corner pixels.

can obtain a variety of different perimeter measurements based on the algorithm used to account for the corner pixels.

Circles: The next level of complexity considered was a series of filled, inscribed, circles. Here again it is easiest to understand how the Image Analysis systems operate by using an inscribed circle having a diameter of four pixels, Figure 6 . When the Euclidean and centroid analysis is used to calculate the maximum Feret's diameters, the results are found from equations 2 and 3 .

$$
\begin{aligned}
& \text { Euclidian Ferret Max }=\sqrt{4^{2}+2^{2}}=4.47 \\
& \text { Centroid Ferret Max }=\sqrt{3^{2}+1^{2}}=3.16
\end{aligned}
$$

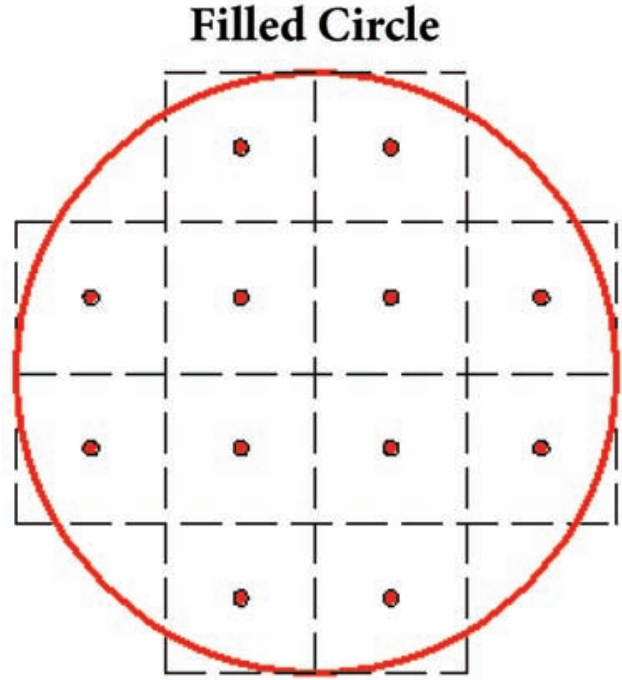

Figure 6. Pixels contained in a filled circle having a diameter of 4 .
In all measurements, the object was found to have an area of 12 square pixels. However, as indicated by the above calculations and measurements, in some cases, the maximum Feret's diameter is larger than the diameter of the inscribed circle, while in the maximum Feret's diameter is smaller than the diameter of the inscribed circle. Based on the other cases, the

number of corners in image, it is not surprising that the perimeter of the inscribed circle has even more variation than the maximum Feret's diameter. As for the rectangles, as the size of the inscribed circles increases, the difference among the parameters measured by the various I/A systems decreases, Figure 7.

For most applications, it is a rare occurrence when the objects to be evaluated are well-defined rectangles that have sides parallel to the principal axes. Generally, an object with curved sides similar to a circle is encountered.

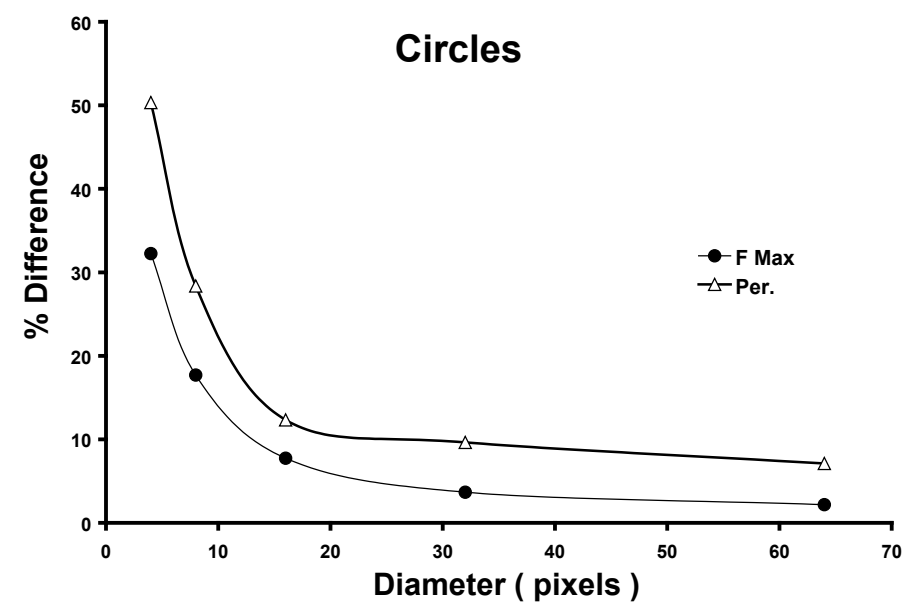

Figure 7. Difference among measured Image Analysis parameters with respect to inscribed circle diameter.

Thus, for practical applications, the results based on the analysis of circular objects best represents what sized objects should be measured if comparisons among different I/A systems are to be made. Hence to have an error of less than 5\%, the minimum feret diameter of the measured objects should be at least 40 pixels. Furthermore, no matter what the size of the object, the differences in perimeter measurements are always greater than the differences in Feret's diameters.

Convex Perimeter: Another perimeter measurement is often discussed; i.e. convex perimeter. In simple terms, it is explained as the perimeter of a rubber band stretched around an object. In most applications for large objects, this may be true; however, this measurement is not quite that simple. In this study, several unusual things were discovered. As before, not all the systems make the measurement in the same manner. In some cases, the perimeter of the rectangles was smaller than the convex perimeter of the rectangles. While, in other cases, the perimeter of the rectangles was larger than the convex perimeter. A very strange occurrence was noted for one of the systems; when the convex perimeter of the rectangles was measured, the pixels at the corners were removed from the rectangle. Thus, the area of the rectangles decreased. This could be a significant problem for irregularly shaped or large features. One other problem was encountered; the freeware did not measure convex perimeter.

Crofton Perimeter: There is another perimeter measurement that was not used since most systems do not include it in the software packages - Crofton Perimeter. The Crofton perimeter is based on intercept counts made by using test lines with a known spacing between the lines. The Crofton perimeter is based on topological relationships that exist between objects and surfaces [2]. In general, the Crofton perimeter is more accurate than the simple perimeter measurements used by most I/A systems.

Shape Factors: With these observations in mind, it is now possible to consider the original problem of nodularity measurements from a more enlightened frame of mind. Through the years, several different shape factors have been proposed by various investigators, as well as manufacturers of I/A systems. Several of the initial shape factors proposed date back to one of the original QTM systems 
create by Cambridge Instruments in the late 1960's [3]. Roundness and shape factor were defined as follows:

$$
\text { Roundness }=\frac{P^{2}}{4 \pi A}
$$

and

$$
\text { S.F. }=\frac{4 \pi A}{P^{2}}
$$

For a circle, each of these parameters is equal to one. As the object becomes more elliptical, the roundness increases, while the shape factor decreases. In time, similar parameters based on convex perimeter were developed. The problem with each of these parameters is that perimeter to the second power is used in each of these formulas. Thus, in evaluation the sphericity of nodularity of a particular object, the square of one of the least accurate I/A parameters is utilized.

ASTM Committee E04 has proposed using a shape factor based on the area of a circumscribed circle as the measurement of the degree of nodularity. For any object that is measured, the objects area and its maximum Feret's diameter are measured. The degree of nodularity is then the area of the measured object divided by the area of the circle that circumscribes it, $\left(\pi F_{\text {Max }}^{2}\right) / 4$. That is,

$$
\text { ASTM Nodularity }=\frac{4 \pi \cdot \text { Area }}{F_{\text {Max }}^{2}}
$$

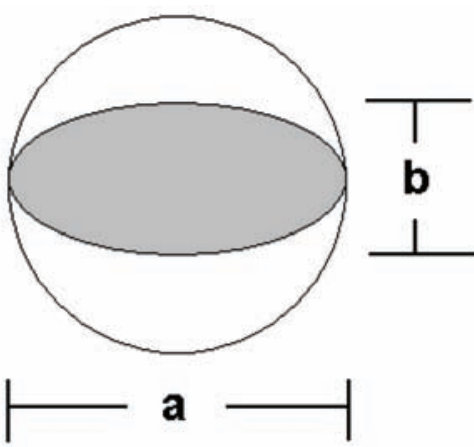

Figure 8. An ellipse, having major and minor axes of $a$ and $b$ respectively, and its circumscribed circle.
This parameter does not contain a perimeter measurement. In addition, it is more sensitive to departures from true circularity than the shape factor previously defined by equation 5 . It is interesting to note that this parameter is referred to as Roundness in the CRC Image Processing Handbook [4]. Consider the relationship between an ellipse and its circumscribed circle, Figure 8.

The area of the circumscribed circle is $\pi \cdot a^{2} / 4$ and the area of the ellipse is Area $=\pi a \cdot(b / 4)$. The approximate perimeter of an ellipse, was first given by Euler [5] as,

$$
\text { Ellipse Perimeter } \approx \pi \sqrt{\frac{a^{2}+b^{2}}{2}}
$$

As the minor diameter of the ellipse decreases, the area of the ellipse decreases in a linear manner as compared to the area of the circle circumscribing it, Figure 9. However, for the term Shape Factor, a significant change in the minor axis of the ellipse must occur before the shape factor begins to correspondingly start to decrease. Thus, the proposed ASTM descriptor of nodularity is a more accurate measuring parameter than the factors including perimeters for two reasons. First, the ASTM parameter is more sensitive to departures from circularity than the shape factor. Second, the linear relationship between the area of the objects means differences in the shape factor at high values are similar to differences in the shape parameter at other values.

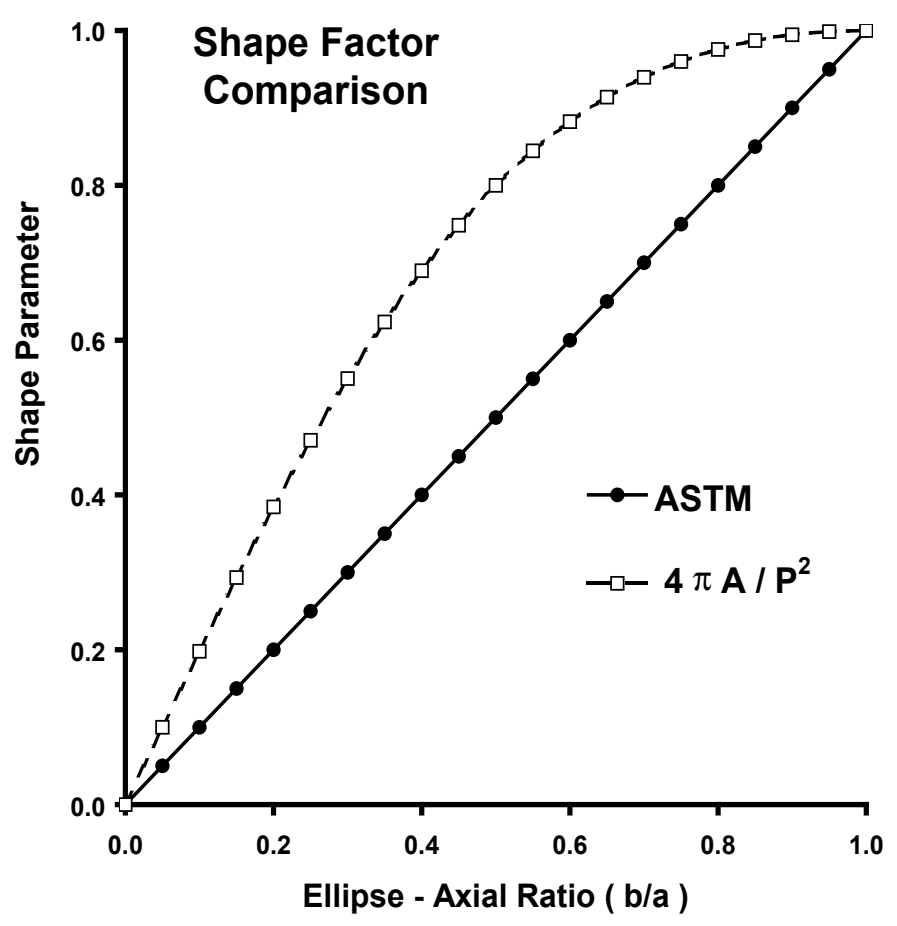

Figure 9. Shape factor comparison.

\section{Conclusions}

Differences among how various I/A systems perform measurements on simple geometric features were clearly established and rationalized. When feature area is measured, all the systems performed the same. However, discrepancies in other parameters such as Feret's diameter and especially perimeter differ quite a lot among the systems evaluated. Convex perimeter was found to be a more complicated parameter than generally perceived, and very system sensitive. For these reasons, shape parameters using any type of perimeter are subject to greater differences among I/A systems than other types of shape parameters. The shape factor proposed by ASTM committee appears to be quite good in describing the degree of roundness of features. For all the parameters considered, the minimum dimension of the objects being measured should be at least 40 pixels to maintain a difference among various systems of $5 \%$ or less.

\section{Acknowledgements}

The assistance of Albert Brandemarte, Gabriel Lucas and Steven Glancy from ASTM Subcommittee E04.14 in performing various tests on the binary images was most helpful. The assistance provided by the various I/A manufacturers at the 2007 M\&M Conference is appreciated.

\section{References}

1. H. E. Exner, 'Analysis of Grain and Particle Size Distributions in Metallic Materials', Int. Met. Reviews, 1972, Review 159, Vol. 17, pp 25-42.

2. M. P. do Carmo, 'Differential Geometry of Curves and Surfaces,' 1976, Prentice Hall, Englewood Cliffs, NJ, pp 41-46.

3. Cambridge Instruments Q720 Instruction Manual, 1969.

4. The Image Processing Handbook, CRC, 2002, Chapter 9.

5. Leonhard Euler, Circa 1773. 


\section{Hitachi High Technologies America}

\section{Achieving Global Excellence Through Strength in Advanced Technologies}
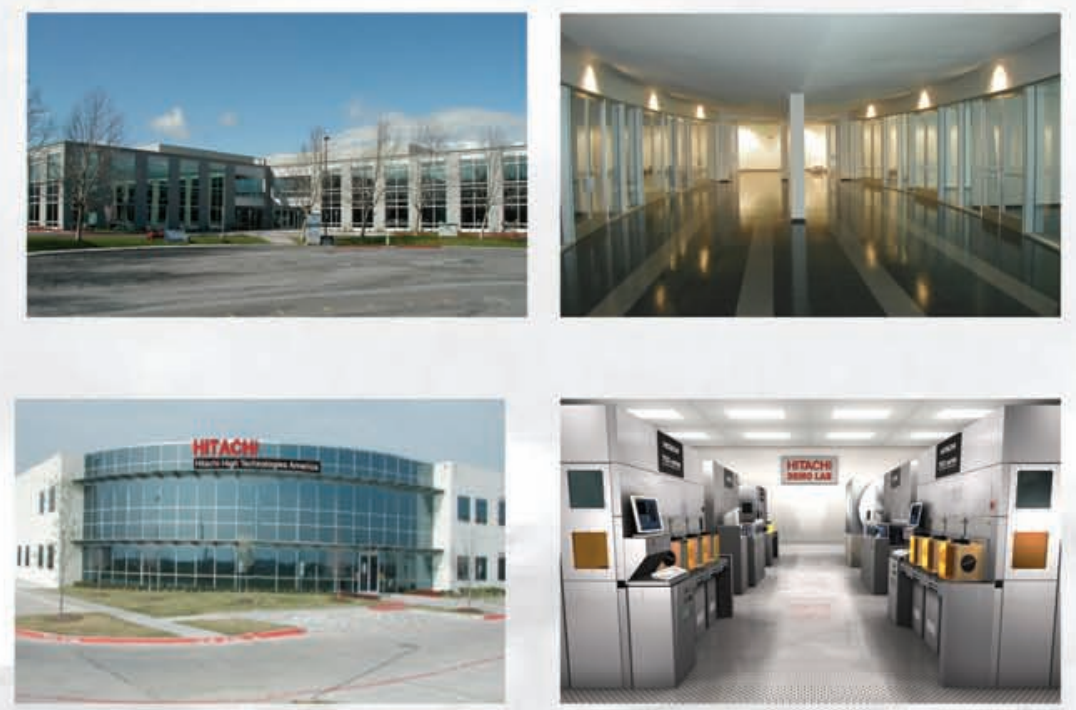

Hitachi High Technologies America - Centers of Excellence Pleasanton, CA (top); Dallas, TX (bottom)

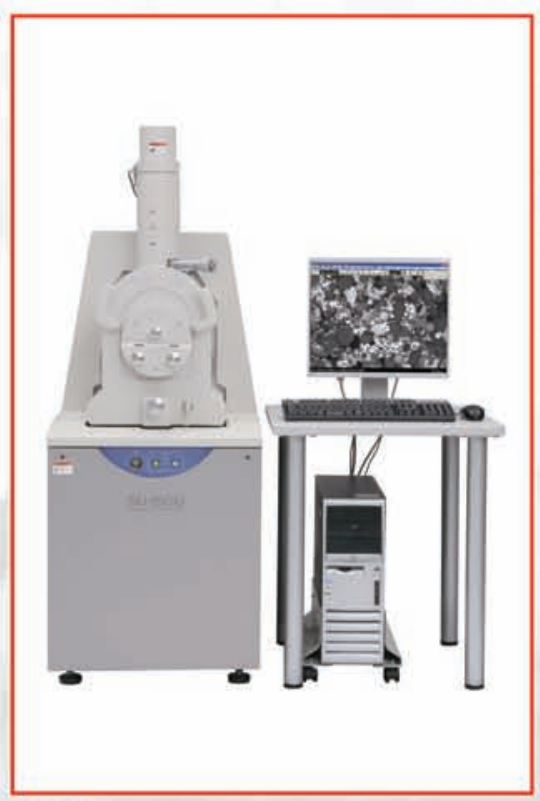

NEW! SU-1500 Scanning Electron Microscope
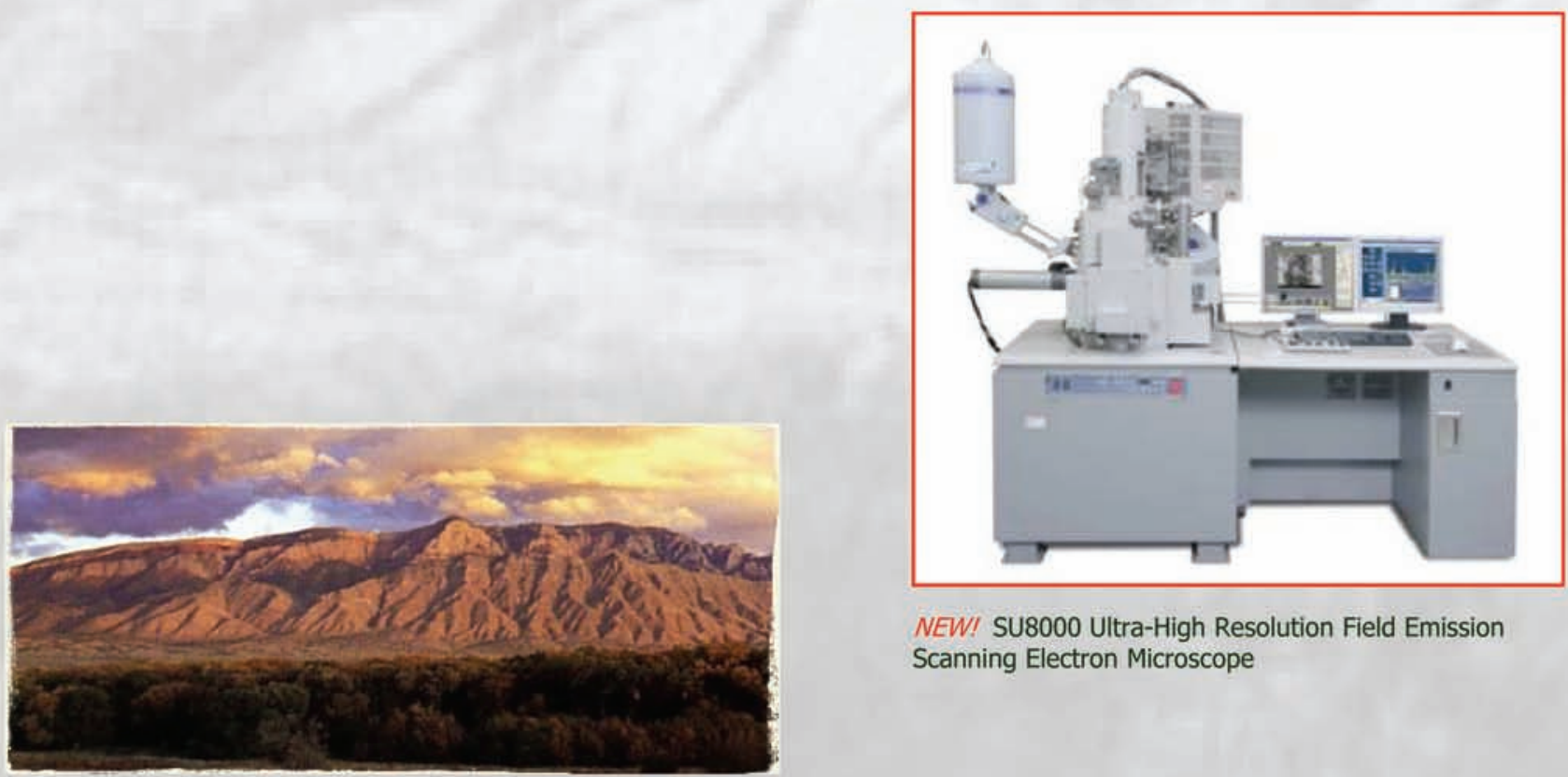

NEW! SU8000 Ultra-High Resolution Field Emission Scanning Electron Microscope

Visit us at M\&M 2008 in Albuquerque, NM Booth \# 1329

Hitachi High Technologies America, Inc. * Nanotechnology Systems Division * 5100 Franklin Drive * Pleasanton, CA $94588925.218 .2800 *$ www.hitachi-hta.com 


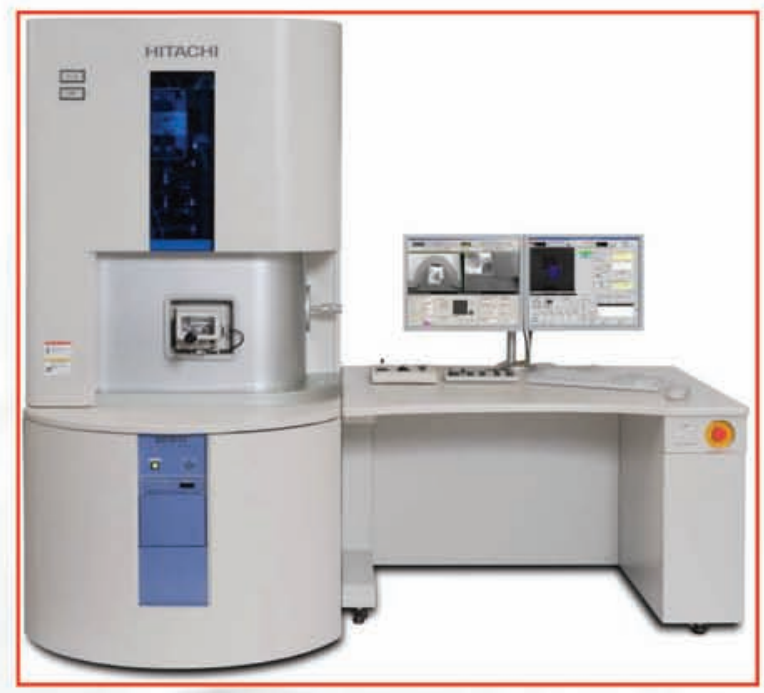

NEW! NB5000 Focused Ion and Electron Beam System

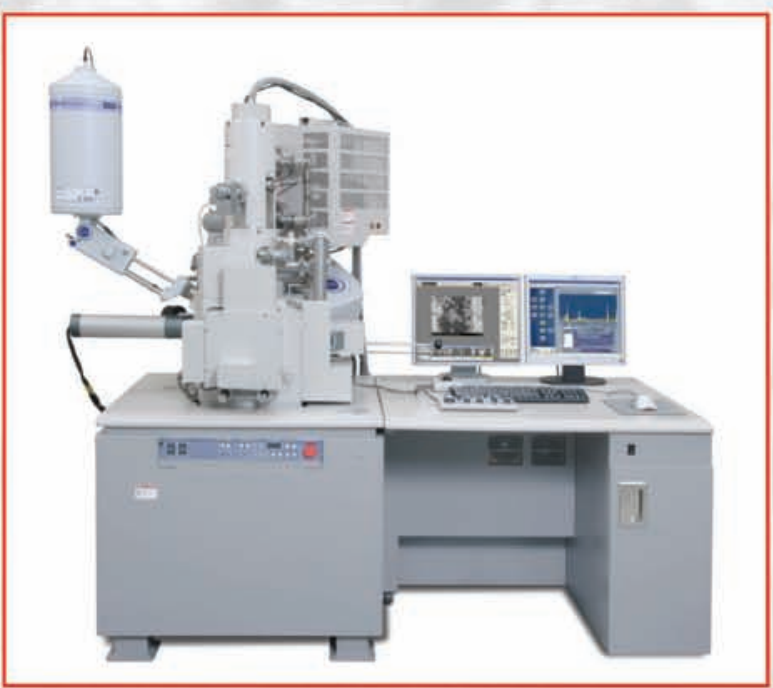

NEW! SU6600 Schottky Emission Variable Pressure Field Emission Scanning Electron Microscope

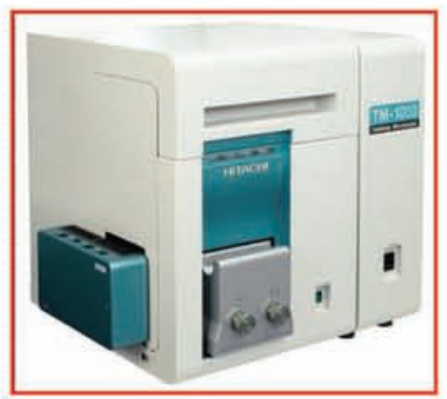

TM-1000 Tabletop Microscope (SwiftEd version)

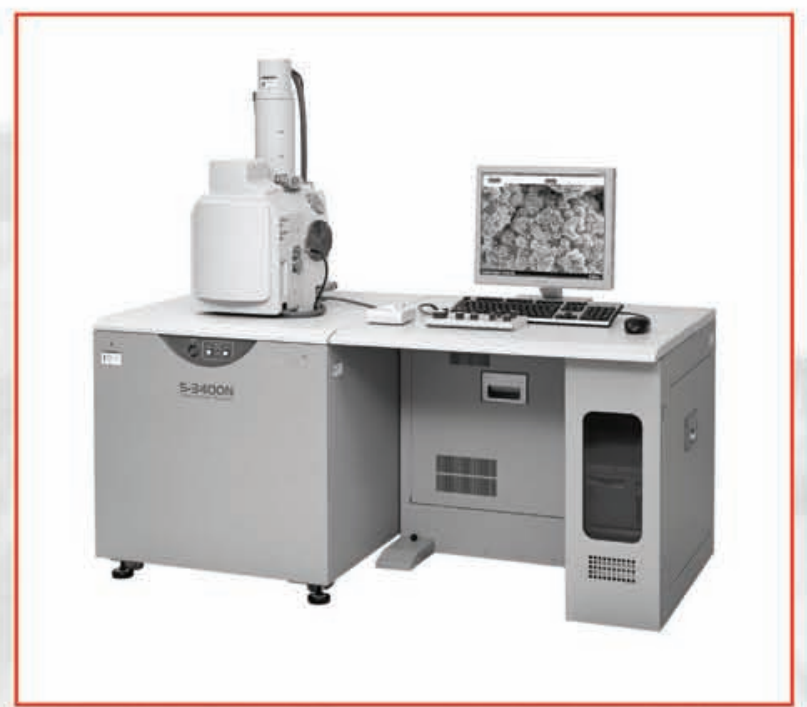

S-3400N Variable Pressure Scanning Electron Microscope

Hitachi will continue to provide the best equipment solutions for our customers and for society by carrying through on our mission. Our corporate statement - Inspire the Next - symbolizes Hitachi's determination and commitment to take on the new challenges of the coming nano era. 